\title{
HOT, WARM, AND COLD CORES: GOLDILOCKS MEETS MASSIVE STAR FORMATION
}

\author{
S. KuRTz \\ Centro de Radioastronomía y Astrofísica, UNAM, Apdo. Postal 3-72, CP 58089 Morelia, Michoacán, Mexico \\ E-mail: s.kurtz@astrosmo.unam.mx \\ (Received October 1, 2004; Accepted December 15, 2004)
}

\begin{abstract}
Molecular clouds present many levels of structure, including clumps and cores of varying size and density. We present a brief summary of these cores, describing their observed physical properties and their place in the star formation process. We conclude with some speculation about pre-proto-stellar stages of molecular cores and the observational challenges in their observation.
\end{abstract}

Key words : stars: formation, ISM: clouds, (ISM:) HII regions, ISM: molecules

\section{INTRODUCTION}

Hot Molecular Cores (HMC) were discovered a little over 10 years ago: R. Cesaroni, C. M. Walmsley and E. Churchwell were the driving force behind the discovery (Cesaroni et al. 1992, 1994). Through a series of ammonia observations using the Bonn 100-meter and the VLA, they confirmed the nature of these objects as hot, dense, compact (i.e., small) molecular condensations. Figure 1 shows an example of the Cesaroni et al. (1994) ammonia $(4,4)$ spectra for the G10.47+0.03 and G31.41+0.31 HMC. About $25 \mathrm{HMC}$ are currently known, with the Orion Hot Core being the best-known example. Typical physical parameters for HMC are listed in Table 1. Worthy of note is the large range of masses - suggesting that there may be more than one class of HMC

About the same time that HMC were being discovered, Plume, Jaffe \& Evans (1992) made a carbon sulfide study of molecular condensations within clouds. The structures they detected are larger and of lower density than HMC, but may be physically important in relation to the HMC, the formation of stellar clusters, and the subsequent evolution of compact HII regions.

A proposed hierarchical scheme showing the structure within molecular clouds and the corresponding HII regions produced by newly born stars is show in Figure 2 (from Kim \& Koo 2001). The smallest, densest condensations - the HMC - are the presumed birth sites of the massive stars. These HMC are embedded within larger, lower density clumps which correspond to the structures reported by Plume et al. (1992). These clumps are embedded within the larger molecular cloud. In this hierarchical structure, a massive star forming inside a HMC can give rise to HII regions on several different size scales as the ionization front propagates through the structural hierarchy of molecular core/clump/cloud.

Proceedings of The 2nd Korea-Mexico Joint Workshop on Physics of the Diffuse Interstellar Medium
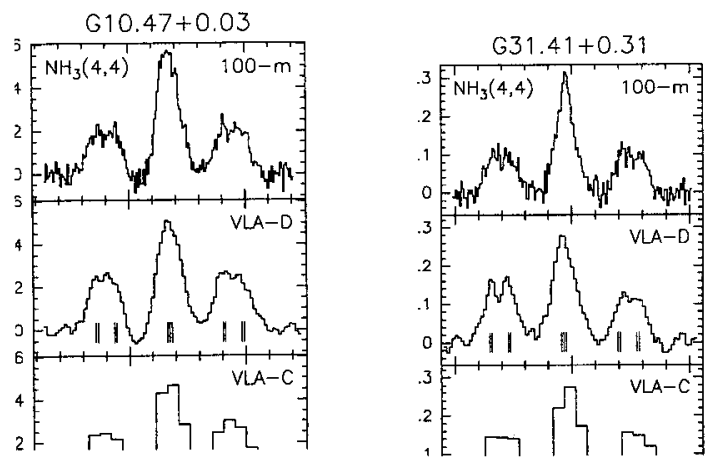

Fig. 1.- Ammonia $(4,4)$ spectra of the G10.47+0.03 and G31.41+0.31 HMC, from Cesaroni et al. (1994). Ammonia $(4,4)$ is a relatively high excitation line, indicating hot molecular gas. That the hyperfine components are seen on either side of the principal line indicates dense molecular gas. The high resolution images with the VLA indicate that it is a compact molecular core.

Theoretical studies of HMC include Kaufman et al. (1998), Osorio et al. (1999), van der Tak et al. (2000), and Nomura \& Millar (2004). These studies examine the possibility of external heating of the cores, and also consider the scenario of a proto-star embedded within the core. In the latter case, a single proto-stellar object is assumed. This is certainly a reasonable starting point for theoretical modeling; nevertheless, we note that the sizes and masses of many HMC suggest that they may form Trapezium-like clusters (see Kurtz 2004).

\section{WARM AND COLD CORES}

The preceding discussion has centered on hot molecular cores. So where do the warm and cold cores come in, and more importantly, why does Goldilocks even 


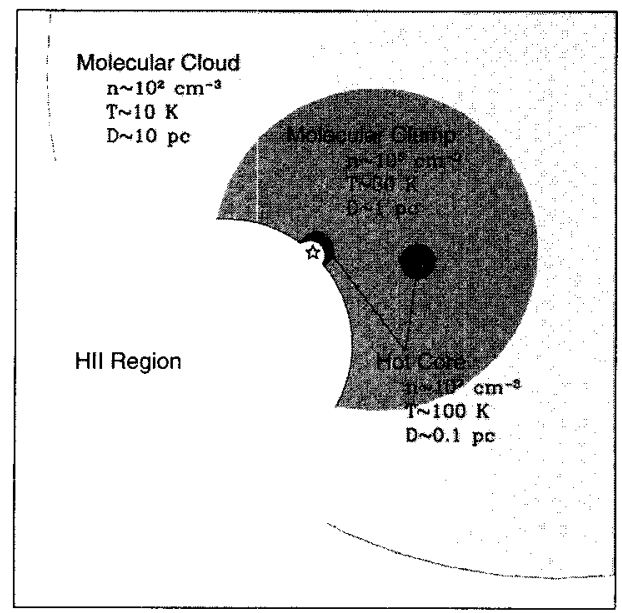

Fig. 2.- A schematic representation showing the hierarchy of structures within molecular clouds. From Kim \& Koo (2001); see also Franco et al. (2000). A massive star, forming within a HMC, may give rise to much more extended structures as it encounters the lower densities of the clump and the cloud.

care what temperature they are?

Although there is not universal agreement on this point, here we have taken hot molecular cores to mean - by definition - molecular cores heated by a luminous, embedded young stellar object (or objects). As such, one might reasonably ask, What sort of entity precedes a hot molecular core? Presumably the answer is a warm molecular core. I.e., a core whose central embedded object is less-evolved, and hence not yet sufficiently luminous to heat the core to $100 \mathrm{~K}$ or more, but only warming it, to a temperature greater than the $\sim 10 \mathrm{~K}$ of the cloud but less than the $\sim 100 \mathrm{~K}$ of the HMC. This simply begs the question, of course, of what entity precedes a warm molecular core? Presumably the answer is a cold molecular core.

The study of these earlier (pre-HMC) phases is particularly important because they may hold the key to distinguishing different modes of high-mass star formation, such as spherical accretion, non-spherical accre-

TABLE 1

Physical Parameters of Hot Molecular CORES

\begin{tabular}{lc}
\hline Parameter & Typical Value \\
\hline Temperature & $>100 \mathrm{~K}$ \\
Density & $>10^{7} \mathrm{~cm}^{-3}$ \\
Diameter & $\lesssim 0.1 \mathrm{pc}$ \\
Luminosity & $\sim 10^{6} \mathrm{~L}_{\odot}$ \\
Mass & $10-10^{4} \mathrm{M}_{\odot}$ \\
\hline
\end{tabular}

tion, coalescence, or some other, as yet un-proposed, model. Moreover, the physical conditions within these cores may determine the IMF of the stellar cluster that will eventually form. Although both warm and cold cores are intrinsically worthy of study, cold cores (being the earlier stage) are perhaps of somewhat greater interest.

As a general scheme, the evolutionary sequence might be imagined to begin with cold cores, lacking any proto-stellar objects, of sizes somewhat smaller than 1 pc, masses of $10^{2}-10^{3} \mathrm{M}_{\odot}$, and temperatures of 10 $20 \mathrm{~K}$. These cold cores would (somehow) form one (or more) proto-stars and eventually become warm molecular cores with sizes of around $0.1 \mathrm{pc}$, masses of $10^{2}-10^{3}$ $\mathrm{M}_{\odot}$, and temperatures of $30-50 \mathrm{~K}$. With further molecular collapse, leading to ever higher optical depths and proto-stellar luminosities, the warm cores would eventually become hot cores, with typical parameters listed in Table 1.

The differences between these high-mass starless (i.e., cold) cores and the low-mass starless cores described elsewhere in this volume by C. W. Lee et al. and by J. Sohn et al. are not clear. High-mass starless cores appear to be up to 100 times more massive than their low-mass counterparts, and this may lead to qualitative differences. Nonetheless, it may prove fruitful to make comparative studies of the two.

Garay et al. (2004) report the detection of four molecular cores in the $1.2 \mathrm{~mm}$ continuum, which do not have IRAS or MSX counterparts. They derive sizes of $0.2-0.3 \mathrm{pc}$, temperatures of $<17 \mathrm{~K}$, densities around $2 \times 10^{5} \mathrm{~cm}^{-3}$, and total masses from 400 to $2,000 \mathrm{M}_{\odot}$. Faúndez et al. (2004) report many additional sources, with typical temperatures of $32 \mathrm{~K}$. These sources may be more representative of warm cores, but they report ten sources with dust temperatures of $25 \mathrm{~K}$ or less, and these may prove to be cold cores. Additional candidates are found in the work of Gibb et al. (2002) and Forbrich et al. (2004). Other workers (e.g., Egan et al. 1998, Molinari et al. 2002, Williams et al. 2004, and Ao, Yang \& Sunada 2004) have probably observed cold and warm cores as well, although additional observations are needed to confirm this.

An intriguing aspect of the cold core candidates found to date is the possibility that they are comprised of distinct populations. Plume et al. (1997), Shirley et al. (2003), and Garay et al. (2004) find linewidths from about $1-10 \mathrm{~km} \mathrm{~s}^{-1}$. If distinct populations of turbulent and non-turbulent cores exist, Will both form proto-stars? Will both form clusters? Will they have the same IMF?

\section{HOW SHOULD WE OBSERVE WARM AND COLD CORES?}

One dilemma for Goldilocks was to find the porridge that was the right temperature. Papa Bear's was too hot, Baby Bear's was too cold, but Mama Bear's was 


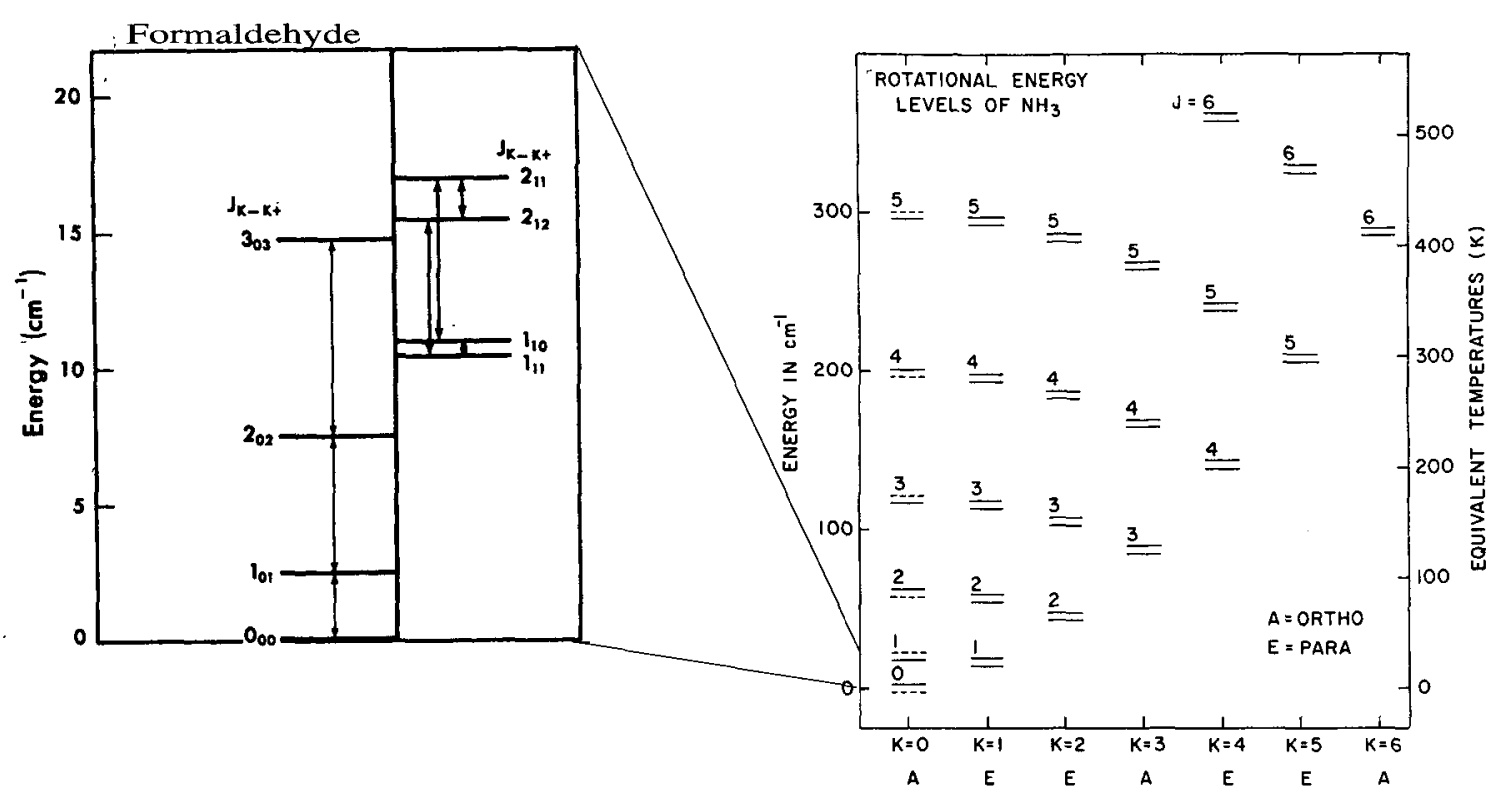

Fig. 3.- Level diagrams for ammonia and formaldehyde (from Ho \& Townes 1983, and Townes \& Cheung 1969, respectively). The ammonia $(1,1)$ transition is at $23 \mathrm{~K}$, the $(2,2)$ at $65 \mathrm{~K}$, and the $(3,3)$ at $125 \mathrm{~K}$. At best, only the $(1,1)$ transition $\%$ would be useful to observe cold cores. Formaldehyde, however, has several transitions at even lower excitation levels.

just right. So what's the right temperature to observe a molecular core? Basically, the answer is hot. Warm cores will present a larger challenge, while cold cores will prove the most difficult to observe.

Hot molecular cores are just right to observe because their high densities and temperatures ensure an active chemistry. The evaporation of grain mantels produces large increases in the gas phase abundances, and coupled with the high temperatures, this provides a wealth of molecular transitions with which to trace the physical and kinematical properties of the gas. Cold cores are just the opposite. Gas phase abundances are much lower because many molecules are frozen out onto grains (e.g., Bergin et al. 1995, 1997; Aikawa et al. 2001,2003 ). At $10-20 \mathrm{~K}$ there is relatively little chemistry going on, and few molecular transitions are excited. Warm cores presumably present an intermediate case.

Ammonia was the first molecular tracer used with HMC, and has been highly successful at probing their inner structure. Other molecules, such as methyl cyanide, methyl acetylene, and carbon sulfide have also been useful probes (e.g., Olmi et al. 2003, Fontani et al. 2002, and Olmi \& Cesaroni 1999). Unfortunately, many of the standard HMC tracers will prove of little or no value for tracing warm - much less cold - molecular cores. The reason is readily evident from figure 3 , which shows the excitation levels for ammonia. The lowest level, $(1,1)$, has an excitation level of $23 \mathrm{~K}$, and hence might be useful to detect the presence of molecular material. But to extract physical parameters, a second transition is needed, and the next higher level,
$(2,2)$ at $65 \mathrm{~K}$ is well beyond the reach of cold (and probably even warm) molecular cores.

Formaldehyde, $\mathrm{H}_{2} \mathrm{CO}$, may be a viable alternative to some of the standard molecular tracers that have been used in HMC. It has transitions at $6 \mathrm{~cm}, 2 \mathrm{~cm}$, and $2 \mathrm{~mm}$, and there are many low-lying levels, in the 10-20 K range (see figure 3). At molecular hydrogen densities below about $10^{5} \mathrm{~cm}^{-3}$ it is super-cooled and seen in absorption (Townes \& Cheung 1969). But at densities above $10^{5} \mathrm{~cm}^{-3}$ the collisional process that populates the lower levels is quenched, and the lines can appear in emission (Garrison et al. 1975). Depletion of formaldehyde may be substantial within pre-protostellar cores (Young et al. 2004) and this may have a significant impact on its use as a cold core tracer. Nevertheless, its potential as a cold core probe is worth exploring.

Additional molecular tracers are possible. Gibb et al. (2002), for example, found that $\mathrm{N}_{2} \mathrm{H}^{+}$was able to trace molecular material that did not appear in $\mathrm{C}^{18} \mathrm{O}$ emission. Methanol also has significant potential (Leurini et al. 2004). Chemical studies of low-mass cores (Bergin \& Langer 1997, Lee et al. 2003) have been very fruitful in identifying useful tracers. Such studies should be extended to high-mass cores as well.

\section{REFERENCES}

Aikawa, Y., Ohashi, N., \& Herbst, E. 2003, ApJ, 593, 906

Aikawa, Y., Ohashi, N, Inutsuka, S., Herbst, E. \& Takakuwa, S. 2001, ApJ, 552, 639 
Ao, Y., Yang, J., \& Sunada, K. 2004, AJ, 128, 1716

Bergin, E. A., \& Langer, W. D. 1997, ApJ, 486, 316

Bergin, E. A., Langer, W. D., \& Goldsmith, P. 1995, ApJ, 441, 222

Cesaroni, R., Churchwell, E., Hofner, P., Walmsley, C. M., \& Kurtz, S. 1994, A\&A, 288, 903

Cesaroni, R., Walmsley, C. M., \& Churchwell, E., A\&A, 256, 618

Egan, M. P., Shipman, R. F., Price, S. D., Carey, S. J., Clark, F. O., \& Cohen, M. 1998, ApJ, 494, L199

Faúndez, S., Bronfman, L., Garay, G., Chini, R., Nyman, L.-A, \& May, J. 2004, A\&A, 426, 97

Fontani, F., Cesaroni, R., Caselli, P., \& Olmi, L. 2002, A\&A, 389, 603

Forbrich, J., Scheyer, K., Posselt, B., Klein, R. \& Henning, Th. 2004, ApJ, 602, 843

Franco, J., Kurtz, S., Hofner, P., Testi, L., GarcíaSegura, G., \& Martos, M. 2000, ApJ, 542, 143L

Garay, G., Faúndez, S., Mardones, D., Bronfman, L., Chini, R., \& Nyman,L.-Å2004, ApJ, 610, 313

Garrison, B. J., Lester, W. A., Miller, W. H., \& Green, S. 1975, ApJ, 200, L175

Gibb, A. G., Wyrowski, F., Mundy, L. G., \& Thompson, M. A. 2002, in ASP Conf. Ser. 267, Hot star workshop III: the earliest stages of massive star birth, ed. P. A. Crowther (San Francisco: ASP), 371

Ho, P. T. P., \& Townes, C. H. 1983, ARA\&A, 21, 239

Kaufman, M. J., Hollenbach, D. J., \& Tielens, A. 1999, ApJ, 497, 276

Kim, K.-T., \& Koo, B.-C. 2001, ApJ, 549, 979

Kurtz, S. 2004, in ASP Conf. Ser. 322, The formation and evolution of massive young star clusters, eds. H.J.G.L.M. Lamers, L.J. Smith \& A. Nota (San Francisco: ASP), 255

Leurini, S., Schilke, P., Menten, K., Flower, D., Pottage, J., \& Xu, L. 2004, A\&A, 422, 573.

Molinari, S., Testi, L., Rodríguez, L. F., \& Zhang, Q. 2002, ApJ, 570, 758

Olmi, L. Cesaroni, R., Hofner, P., Kurtz, S., Churchwell, E., \& Walmsley, C. M. 2003, A\&A, 407, 225

Olmi, L., \& Cesaroni, R. 1999, A\&A, 352, 266

Osorio, M., Lizano, S., \& D'Alessio, P. 1999, ApJ, 525, 808

Plume, R., Jaffe, D. T., \& Evans, N. J. 1992, ApJS, 78, 505

Plume, R., Jaffe, D. T., Evans, N. J., Martín-Pintado, J., \& Gómez-González, J. 1997, ApJ, 476, 730

Nomura, H. \& Millar, T. J. 2004, A\&A, 414, 409

Shirley, Y., Evans, N. J., Young, K. Knez, C., \& Jaffe, D. T. 2003, ApJS, 149, 375
Townes, C. H., \& Cheung, A. C. 1969, ApJ, 157, L103 van der Tak, F., van Dishoeck, E., Evans, N. J., \& Blake, G. 2000, ApJ, 537, 283

Williams, S. J., Fuller, G. A., \& Sridharan, T. K. 2004, A\&A, 417, 115

Young, K. E., Lee, J.-E., Evans, N. J., Goldsmith, P. F., \& Doty, S. D. 2004, ApJ, 614, 252 